\title{
Evaluation of disruption/permeabilization methodologies for Microcystis aeruginosa as alternatives to obtain high yields of microcystin release
}

\author{
Pedro Geada $^{\mathrm{a}, *}$, Luís Loureiro ${ }^{\mathrm{a}}$, José A. Teixeira ${ }^{\mathrm{a}}$, Vítor Vasconcelos ${ }^{\mathrm{b}}$, António A. Vicente ${ }^{\mathrm{a}}$, \\ Bruno D. Fernandes ${ }^{\mathrm{a}}$ \\ ${ }^{\text {a }}$ CEB-Centre of Biological Engineering, University of Minho, Braga, Portugal \\ ${ }^{\mathrm{b}}$ CIIMAR/CIMAR - Interdisciplinary Centre of Marine and Environmental Research and Department of Biology, Faculty of Sciences, University of Porto, Porto, Portugal
}

\section{A R T I C L E I N F O}

\section{Keywords:}

Microcystin

Intracellular organic matter

Cell disruption

Microcystis aeruginosa

Downstream optimization

\begin{abstract}
A B S T R A C T
The extraction of the wide range of useful bioactive compounds produced by cyanobacteria is still a major bottleneck at industrial scale. In addition to the high costs, extraction efficiencies are also commonly low, with low cell disruption efficiencies playing a particularly significant role in intracellular compounds' release. To increase the chances of an extended use of the cyanobacteria toxin microcystin in several biotechnological fields, we aimed to optimize five different disruption techniques: bead milling, microwave, freeze-thaw cycles, highspeed homogenization, and sonication. For each of the methods tested, the conditions that maximized the intracellular organic matter release were: i) $20 \%$ of beads and treatment time of $7 \mathrm{~min}$ (bead milling); ii) $800 \mathrm{~W}$ for $1.5 \mathrm{~min}$ (microwave); iii) three 12 -h freeze-thaw cycles at $-20^{\circ} \mathrm{C}$; iv) $15,000 \mathrm{rpm}$ for $7 \mathrm{~min}$ (high-speed homogenization); and v) $40 \mathrm{kHz}$ for $10 \mathrm{~min}$ (sonication).

Sonication and freeze-thaw cycles followed by sonication revealed to be the most effective methodologies to ensure a maximum intracellular organic matter release and, consequently, microcystin availability for being extracted. The decrease of cells' viability was however more evident in freeze-thaw cycles, freeze-thaw cycles followed by sonication, and microwave where only $0.3,0.05$ and $0.9 \%$ of the initial cells, respectively, maintained their viability after being treated. On the other hand, sonication and bead milling reduced the viability of the original culture to 5 and $15.5 \%$, respectively, while high-speed homogenization did not show any significant differences compared to control.

According to the results obtained in this study, the most suitable methodology to maximize the release of microcystin was therefore the use of sonication $(40 \mathrm{kHz})$ during $10 \mathrm{~min}$.
\end{abstract}

\section{Introduction}

Cyanobacteria, also known as blue-green algae, are ancient photosynthetic microorganisms whose importance is far beyond the fact of probably being responsible for the origin of oxygen on Earth's atmosphere billion years ago [1]. This group of Gram-negative prokaryotes is nowadays scattered around the planet due to the diversity displayed, as well as the ability to continuously and quickly adapt to their surrounding environment, such as increasing temperatures. Several cyanobacteria species are well-known bloom-forming microorganisms some of them are also toxin-producers - generally associated with water bodies' eutrophication as a consequence of the intense anthropogenic activity [2].

Similarly to microalgae, cyanobacteria produce important secondary metabolites (including vitamins, toxins, enzymes and pigments) and consequently represent a tremendous source of potential high added-value compounds to apply in food, feed, pharmaceutical, chemical, and biofuels sectors [3,4].

Microcystis aeruginosa, one of the most studied cyanobacteria, is frequently cited as an environmental hazard that might present a serious threat for humans [5]. The authorities' concern derives from the ability of certain strains of $M$. aeruginosa to produce potent toxins such as microcystins (MC), which are hepatotoxins responsible for human deaths and illnesses episodes [3,6]. Nevertheless, numerous studies point this cyanobacterium as a source of a significant range of bioproducts or even as an added-value itself by integrating this species in some industrial processes [7-11]. Despite MCs are continuously labelled as negative for health, multiple possible applications have been unveiled by studies performed throughout the years. The use as pure standards for human and environmental risk assessment tests, as tool

\footnotetext{
* Corresponding author.

E-mail address: pedrogeada@ceb.uminho.pt (P. Geada).
} 
for molecular and cell biology studies, as antialgal agent or as active principle in anticancer drugs production, are just some the potential applications of these cyanotoxins [3].

In spite of the rising interest originated by recent findings, most of these compounds (including MC) are intracellular and cell rupture techniques are required, commonly representing a major bottleneck at industrial scale [3]. Taking the example of microalgae, a wide range of disruption methods is available to be applied on cyanobacteria cultures. Generally, these downstream processes can be divided into two distinct groups: mechanical (e.g. microwave, bead milling, ultrasonication) and non-mechanical (e.g. chemical, enzymatic) methods [12]. In spite of the variety of effective techniques, there is a need for mild, inexpensive, and low-energy consumption methodologies to meet the exploitation of more unstable cyanobacterial metabolites. The conventional disruption methods usually utilize chemicals or very high pressure optimized conditions to obtain a given product, but might result in severe damage for many other products [13]. The use of mild-processing techniques such as pulsed electric fields, enzymes, and ultrasonication - might represent an appropriate alternative to overcome this major bottleneck $[12,13]$. Although several studies have already been published about the disruption of $M$. aeruginosa cells, there is a lack of a thorough comparison between the disruption efficiency of the methods and their impact on cells and products of interest [14-17]. Additionally, harsh conditions are frequently applied in downstream processes since complete removal of both cells and secondary metabolites, namely MCs, is the main goal - especially when wastewater treatment plants are considered. However, bearing in mind the potential applications of MC, the purpose of this work is to achieve very high disruption efficiencies without compromising the stability of the cyanotoxin (i.e. operating under mild and effective conditions). In this sense, we propose to optimize a series of disruption methods - bead milling, freeze-thaw cycles (FTC), microwave, sonication, and high-speed homogenization (HSH) and compare them in terms of disruption efficiency and MC and intracellular organic matter release (IOMR).

\section{Materials and methods}

\subsection{Microorganism and culture conditions}

Cyanobacterium Microcystis aeruginosa LEGE 91094 utilized in this study was kindly provided by the Interdisciplinary Centre of Marine and Environmental Research (CIIMAR - Porto, Portugal) collection (LEGE CC) and maintained in $\mathrm{Z8}$ medium [18] under $10 \mu \mathrm{mol}_{\text {photons }} \cdot \mathrm{m}^{-2} \cdot \mathrm{s}^{-1}$ using a $12: 12 \mathrm{~h}$ light-dark cycle at room temperature. Stock cultures were renewed on a monthly basis.

\subsection{Disruption techniques}

Five different mild disruption methods were performed and compared: $i$ ) bead milling; ii) microwave; iii) freeze-thaw cycles (FTC); $i v$ ) high-speed homogenization (HSH); and $v$ ) sonication. These methods and their application conditions were chosen specifically to prevent damages in microcystins' structure $[19,20]$, thus avoiding the need to check the occurrence of eventual structural alterations by expensive analytical techniques such as LC-MSMS. Tests were made in triplicate using $25 \mathrm{~mL}$ of a culture of $M$. aeruginosa presenting a biomass concentration of approximately $0.5 \mathrm{~g} \cdot \mathrm{L}^{-1}$.

\subsubsection{Bead milling}

Bead milling assays were carried out using glass beads of small diameter $(149-250 \mu \mathrm{m})$ and a vortex mixer Clifton Cyclone CM-1 (Nickel Electro Ltd., UK). Several bead ratios (between 0 and $80 \%$ of the culture volume) and treatment time intervals (1-10 min) were tested.

\subsubsection{Microwave}

Cell disruption caused by microwave treatment was conducted in a
Speedy $21 \mathrm{~L}$ microwave oven (Taurus, Spain) varying the time $(0.5-2 \mathrm{~min})$ and the power (Defrost $(<400-800 \mathrm{~W})$. To avoid overheating and intracellular content release caused by temperature (above $60{ }^{\circ} \mathrm{C}$ - data not shown), short-period cycles were applied for each power used (four $30 \mathrm{~s}$ cycles for defrost; eight $15 \mathrm{~s}$ cycles for the remaining power intensities) and samples were kept in ice between cycles until room temperature (roughly $20^{\circ} \mathrm{C}$ ) was reached.

\subsubsection{Freeze-thaw cycles (FTC)}

The effect of FTC was tested at two different temperatures $(-20$ and $-70{ }^{\circ} \mathrm{C}$ ). The number of cycles to apply was also assessed. Since the defrosting process using hot water led to release of intracellular material (data not shown), it was done placing tubes containing frozen samples on a vessel with tap water.

\subsubsection{High-speed homogenization (HSH)}

A T-25 digital Ultra-Turrax ${ }^{\circledR}$ (IKA ${ }^{\circledast}$, Germany) was utilized to perform cell disruption experiments under $\mathrm{HSH}$ conditions. The influence of three distinct agitation speeds $(10,000,15,000$ and 20,000 rpm) and treatment time (1-15 min) was determined.

\subsubsection{Sonication}

Sonication was performed using an ultrasonic processor VCX 500 (Sonics \& Materials, Inc., USA) and subjecting cells to different frequencies (20 and $40 \mathrm{kHz}$ ) during certain periods of time (1-15 min). Cycles applied consisted of $3 \mathrm{~s}$ of treatment on and $9 \mathrm{~s}$ off. The culture was continually kept in ice to prevent overheating.

\subsection{Cell disruption efficiency analysis}

The cell disruption methods were compared using three different techniques: i) flow cytometry; ii) IOMR; and iii) toxin quantification.

\subsubsection{Flow cytometry}

Flow cytometry analysis was performed using an $\mathrm{EC} 800^{\mathrm{TM}}$ flow cytometer analyzer (Sony Biotechnology Inc., USA). A total of 50,000 events were collected for each triplicate of the samples in order to present reasonable confidence intervals of cell concentrations and consistent fluorescence results. Red fluorescence signals, which are associated with the total chlorophyll content, were collected by a $665 \mathrm{~nm}$ long-pass filter, FL3. The number of events and fluorescence signals were evaluated through the EC800 1.3.6 analysis software (Sony Biotech) and the Flowing software 2.5.1. Cell disruption efficiency results obtained by flow cytometry were compared using the methods described in Günerken et al. [21]. Briefly, the direct cell counting data was one of the methods used to evaluate the disruption efficiency; the other method consisted in splitting the histograms of FL3-Peak-Lin (radius of spherical cells) and FL3-Lin (area) into quadrants, corresponding the upper right quadrant to the healthy population of microalgae.

\subsubsection{IOMR measurement}

After disruption treatment, samples of $500 \mu \mathrm{L}$ were taken and then centrifuged at $12,000 \mathrm{rpm}$ for $10 \mathrm{~min}$. The supernatant was collected and its absorbance was measured at a wavelength of $254 \mathrm{~nm}$ in a Synergy ${ }^{\mathrm{TM}}$ HT Multi-Detection Microplate Reader (BioTek Instruments, Inc., USA). This procedure allowed detecting the IOMR from the cells at different conditions [22]. The extracellular organic matter present in original sample (before starting the disruption process) was also determined applying the same method. To facilitate the interpretation of results, an IOMR factor was calculated for each sample tested according to the following equation:

IOMR factor $=\frac{\left(\mathrm{Abs}_{\mathrm{t}}-\mathrm{Abs}_{0}\right)}{\mathrm{Abs}_{0}}$

where $\mathrm{Abs}_{\mathrm{t}}$ refers to the absorbance measured at $254 \mathrm{~nm}$ after 
disruption treatment during a certain period of time (t, min) and $\mathrm{Abs}_{0}$ is the absorbance measured at $254 \mathrm{~nm}$ of the original sample (before treatment).

\subsubsection{Toxin quantification}

The Microcystins-ADDA ELISA Kit (Abraxis, Inc., USA) was used to determine the concentration of total MC toxin before and after cells having been treated. The amount of MC in each sample was obtained following the instructions of the Microcystins-ADDA ELISA Kit. Samples were diluted according to the manufacturer's recommendations and the absorbance was measured at $450 \mathrm{~nm}$ using a Synergy ${ }^{\mathrm{TM}}$ HT MultiDetection Microplate Reader (BioTek Instruments, Inc., USA).

\subsubsection{Statistical analysis}

The experimental data were analysed through one-way ANOVA followed by a post hoc Tukey test using Statistica 10.0.228.8 software (Statsoft Inc., USA).

\section{Results and discussion}

\subsection{Optimization of cell disruption techniques}

The comparison of all tested techniques was divided into two major steps: optimization of each disruption method mainly considering the IOMR during the process (optimal conditions are presented in Table 1) and further comparison of optimal conditions of each strategy by measuring the disruption efficiency and organic matter and toxin release (Section 3.2).

\subsubsection{Bead milling}

The first stage of bead milling process characterization was developed by comparing the effect of different beads relative percentages (between 0 and $80 \%$ of the culture volume) at a fixed treatment time of 5 min (data not shown). The use of a relative percentage of glass beads around $20 \%$ was found to cause the highest release of IOM and thus to be the most efficient ratio causing cell disruption/permeabilization. Taking into account this information, it was decided to fix this percentage of beads for the following evaluation tests on the influence of bead milling treatment time (between 1 and $10 \mathrm{~min}$ ) - data not shown. The filling ratio employed here as being the optimal condition (Table 1) is considerably lower when compared to microalgae processing information sources stating that beads' volume should be kept between 50 and $90 \%$, typically being $80-85 \%$ [23]. Our results also showed a different behaviour in terms of the disruption efficiency with bead load variation, since some authors observed higher cell disintegration yields when increasing ratios are applied [24,25]. However, these differences might be explained by the use of different beads (in terms of size and composition), cell concentration, and microorganisms, which affect significantly the process' effectiveness.

The influence of treatment time on cell disruption using bead

Table 1

Optimal operating conditions of all disruption techniques tested (bead milling, microwave, FTC, HSH, and sonication) with the respective parameters evaluated and efficiencies obtained (by means of IOMR factor).

\begin{tabular}{lccc}
\hline Disruption method & Parameter 1 & Parameter 2 & Efficiency \\
\hline \multirow{2}{*}{ Bead milling } & Bead $\%(\mathrm{v} / \mathrm{v})$ & Time $(\mathrm{min})$ & IOMR factor \\
& 20 & 7 & $1.1 \pm 0.0$ \\
Microwave & Power $(\mathrm{W})$ & Time $(\mathrm{min})$ & IOMR factor \\
& 800 & 1.5 & $0.9 \pm 0.0$ \\
FTC & Temperature $\left({ }^{\circ} \mathrm{C}\right)$ & Number of cycles & IOMR factor \\
& -20 & 3 & $4.3 \pm 0.1$ \\
HSH & Speed $(\mathrm{rpm})$ & Time (min) & IOMR factor \\
& 15,000 & 7 & $0.4 \pm 0.1$ \\
Sonication & Frequency $(\mathrm{kHz})$ & Time (min) & IOMR factor \\
& 40 & 10 & $3.5 \pm 0.1$ \\
\hline
\end{tabular}

milling technique was evaluated and the highest IOMR was detected after $7 \mathrm{~min}$ (Table 1). Generally, increasing cell disintegration is expected with treatment time extension until it reaches a maximum owing to complete disruption of microorganisms, which seems to be the case. The optimal duration for liberation of IOM from $M$. aeruginosa applying the bead milling technique $-7 \mathrm{~min}$ - is in agreement with other studies performed using distinct microalgae, where a period between 4 and 9 min was considered to be the best treatment time for cell disruption and, consequently, high-value metabolites' release [26,27].

\subsubsection{Microwave}

Microwave effectiveness on cell disruption was assessed by testing four different power intensities (defrost $(<400,400,600$, and $800 \mathrm{~W}$ ) following the IOMR during $2 \mathrm{~min}$ (data not shown). Generally, $800 \mathrm{~W}$ power intensity treatments revealed higher efficiency for all time intervals measured, resulting in higher IOMR of $M$. aeruginosa cells. Subjecting cyanobacteria to a 1.5 min treatment using this power intensity was considered the optimal condition to apply microwave as a disruption/permeabilization technique (Table 1). Comparing with other studies, high disruption (approximately $94 \%$ ) and oil extraction yields were obtained in tests performed at $80-95^{\circ} \mathrm{C}$ for periods of time ranging from 20 to $30 \mathrm{~min}[28,29]$. However, our goal was to assess the efficiency of microwaves only and avoid possible interference of temperature and solvents that would possibly increase IOMR effectiveness. Additionally, metabolites' denaturation is frequently observed as a consequence of increasing temperature, which should be prevented as well. Despite of the significant effect of long-lasting microwave treatments, Balasubramanian et al. [28] also found that there is no direct relationship between treatment time and extraction efficiency, since extraction yields of some algae oils were higher for shorter periods of time and then decreased when extended treatment times were applied. Thus, optimal extraction conditions are deeply dependent on the type of metabolite targeted.

\subsubsection{Freeze-thaw cycles (FTC)}

Freeze-thaw methodology showed higher IOMR from M. aeruginosa cells using three $12 \mathrm{~h}$ freezing cycles at $-20^{\circ} \mathrm{C}$ (Table 1). This is in agreement with the common use of this cell disruption technique since many ELISA quantification kits, for instance, have manufacturers' instructions pointing to the use three freeze-thaw cycles as a means to cause rupture of the cells. This method takes advantage of ice crystals formation inside cells to damage cell wall and release intracellular compounds. The duration of freezing cycles can however vary significantly, being in some cases as short as $2 \mathrm{~h}$ [15].

\subsubsection{High-speed homogenization (HSH)}

HSH tests were carried out up to 10 min under 10,000, 15,000, and $20,000 \mathrm{rpm}$ (data not shown). The use of a stirring velocity of $15,000 \mathrm{rpm}$ frequently revealed higher release rates of IOM throughout the disruption treatment (Table 1). According to the results obtained, the release of intracellular compounds applying this speed is maintained constant from $7 \mathrm{~min}$ onwards (data not shown). Consequently, this might be considered the most suitable time to operate when $M$. aeruginosa cells disruption is intended via HSH. This mechanical method is frequently applied to extract compounds of interest from a number of microorganisms - as the case of microalgae [12]. Although the process may, in some cases, be as short as $1 \mathrm{~min}$ (or even less) $[30,31]$ due to the combination of hydrodynamic cavitation and solvents, rotation speed is commonly set in the range 10,000 to $14,000 \mathrm{rpm}$, which is close to the value achieved in this study (Table 1). When solvents are not part of the extraction process, treatment duration can, however, be longer ( $6 \mathrm{~min}$ ) and approach our optimum [32]. Nevertheless, the agitation rate applied by Sun et al. [32] was considerably higher than $15,000 \mathrm{rpm}$. 


\subsubsection{Sonication}

By comparing both frequencies tested, it is possible to infer that sonication performed at $40 \mathrm{kHz}$ always presented better disruption/ permeabilization yields, regardless of the treatment time (data not shown). Additionally, these data also showed that maximum IOMR was attained after $10 \mathrm{~min}$ (Table 1). Despite of being widely used for the disruption of microalgae and subsequent extraction of added-value products [12,33], (ultra)sonication process optimization is deeply associated with the microorganism under study [34], the metabolite(s) of interest to be recovered and whether solvents are part of the process or not [35]. In the specific case of $M$. aeruginosa, the optimal treatment time presented in Table 1 is in agreement with the procedure implemented by other authors such as Pestana et al. [15], although the frequency applied in that study is just $50 \mathrm{~Hz}$. Regarding the other parameter studied, $\mathrm{Wu}$ et al. [17] compared the inactivation of $M$. aeruginosa using low frequency $(20 \mathrm{kHz})$ and high frequency $(580$ and $1146 \mathrm{kHz}$ ) sonication concluding that lower frequencies are more effective than higher ones.

\subsection{Comparison of cell disruption techniques}

\subsubsection{Impact on cells}

The optimal conditions of each disruption method were selected and a new experiment was performed where all techniques were compared. Since sonication and FTC were found to promote the highest rates of IOMR individually, an additional test was performed using FTC as pretreatment of sonication. The number of absolute counts and, among these, the corresponding number of cells that remained viable after applying the disruption methods, are presented in Fig. 1.

According to these results it is possible to verify that, after the treatments, most of the absolute counts obtained do not correspond to the region of $M$. aeruginosa cells. The only exception seems to be the HSH since it presents a similar number to the control, indicating that only a few cells were affected by this technique. Sonication and FTC followed by sonication appear to be highly destructive processes and to reduce the debris of cells in such a way that a great part of them is not even accounted by the cytometer (Fig. 1). Despite of the effectiveness shown by sonication, approximately $5 \%$ of the initial culture survived to this method. Differently, the number of cells that survive to FTC followed by sonication can be neglected once it represents just $0.05 \%$ of the original amount of biomass. As opposed to sonication, bead milling, and particularly microwave and FTC methods, present a high number of absolute counts, but the number of viable cells found in the culture is significantly lower (Fig. 1). Although FTC and microwave methods

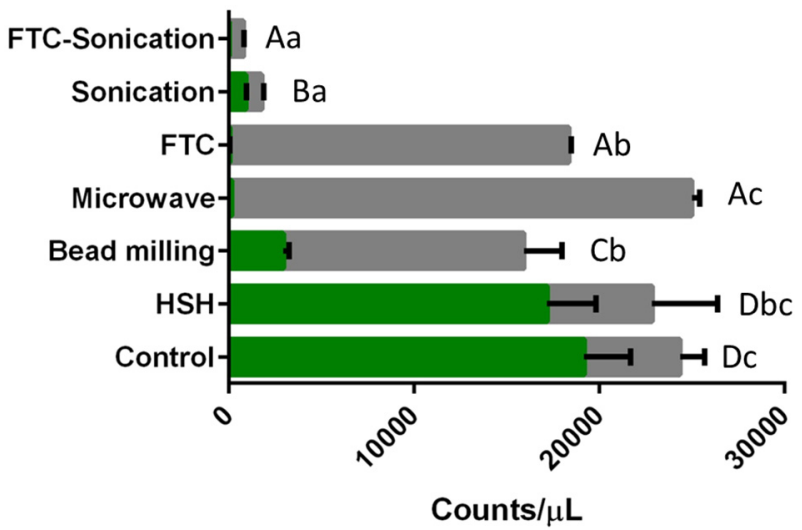

Fig. 1. Quantification of the absolute counts $(\square)$ and surviving $M$. aeruginosa cells counts $(\square)$ by flow cytometry after applying the different disruption methods. Error bars represent the standard deviation for three experiments. According to Tukey's test, the methods presenting the same letter (uppercase letters for surviving cells and lowercase letters for absolute counts) do not differ significantly ( $\alpha=0.05)$. seem not to be as aggressive to cells as sonication (the number of absolute counts is considerably greater), a higher impact on cells' viability was obtained (99.7 and $99.1 \%$, respectively, of the initial sample was damaged and did not keep viable). Regarding bead milling, the number of viable cells found in culture after treatment was around $15.5 \%$.

These results seem not to be in agreement with some other studies since sonication is commonly pointed as an inefficient disruption method [29,36]. For instance, the difference between the disruption efficiency obtained for sonication and microwave was reported as high as $27.2 \%$ by McMillan et al. [29], which is clearly not the case in our results. However, this disparity may be due to the species-dependency of disruption effectiveness once most of the studies use $C$. vulgaris $[29,36]$, commonly considered a more robust and difficult-to-break microorganism.

By evaluating the fluorescence of the cells that were observed in treated samples (Fig. 1), we were able to get interesting information about the effect of each disruption technique over $M$. aeruginosa (Fig. 2).

Through these data, a more linear profile of fluorescence is observed for sonication and HSH, suggesting that for these treatments most of the cells kept a cellular structure which is more similar to that of cells in control samples. This means that sonication and HSH provide more uniform treatment conditions in the whole volume of the samples to which they are applied. However, the majority of the absolute counts detected after HSH (Fig. 1) is placed in the upper right quadrant, while in the case of sonication only about half of them are in that quadrant (Fig. 2). This means that, from the number of absolute counts obtained after treatment, almost all of them are cells that survive to $\mathrm{HSH}$ and keep their viability, whereas only circa $52 \%$ have shown the same behaviour under sonication.

However, the number of absolute counts after sonication is considerably lower than $\mathrm{HSH}$, as shown in Fig. 1. Although the profiles exhibited by cells treated with FTC and bead milling generally show a linear fluorescence profile, it is possible to see some counts displaced and scattered throughout the lower left quadrant. The impact on cells' viability is though distinct in these two methods. The $15.5 \%$ of cells that survived to bead milling represent approximately $32 \%$ of the absolute counts, whilst the use of FTC left $<0.4 \%$ of cells with viability. Consequently, several counts are observed in the upper right quadrant of the bead milling and very few appear there in the case of FTC. In spite of being slightly less efficient than FTC, microwaves $(0.9 \%$ of the cells survived to the method, Fig. 1) do not present any trend on fluorescence profile, which is associated with a great variability in terms of cells' size and shape. The dispersity observed in the lower left quadrant of FTC, bead milling and especially microwave (Fig. 2), indicates a random effect over cells and it makes sense considering that the processing involving these three methodologies is not as constant and uniform as in the case of sonication and HSH. Heo et al. [36] have verified the same behaviour applying microwave irradiation and sonication to $C$. vulgaris. According to those authors, cells' membranes were severely damaged when microwave was utilized, whereas sonication was responsible for cracking or tearing up the cells. Furthermore, it was also concluded that microwave irradiation treatment was not able to disrupt cells evenly [36]. Regarding the case of FTC followed by sonication, it is possible to see a conjugation of both individual profiles of each treatment; however, the predominant effect seems to be induced by sonication (Fig. 2).

\subsubsection{Organic matter release}

Besides the damage inflicted to $M$. aeruginosa by the optimized technologies, another parameter was also evaluated - the IOMR (Fig. 3).

Fig. 3 shows that the rate of organic matter released by cells was considerably promoted by sonication, being the FTC responsible just for a slight increment when applied as pre-treatment. The IOMR caused by microwave irradiation was surprisingly low in spite of being one of the 
a

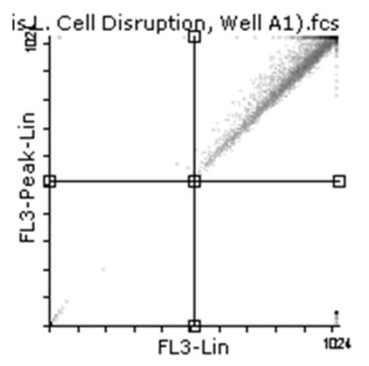

d

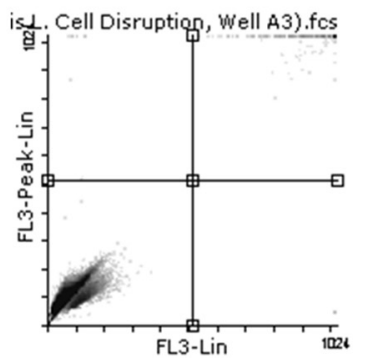

b

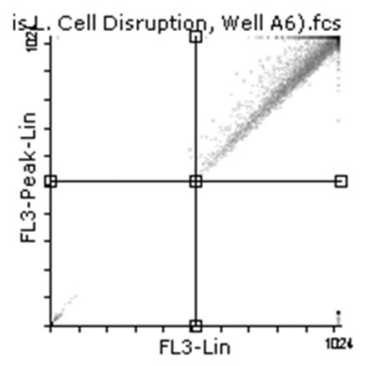

e

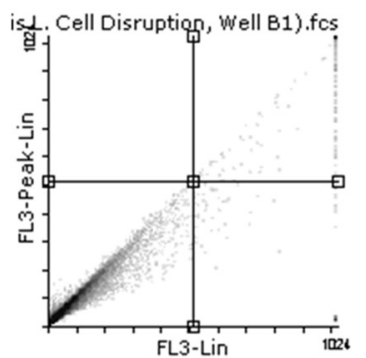

C

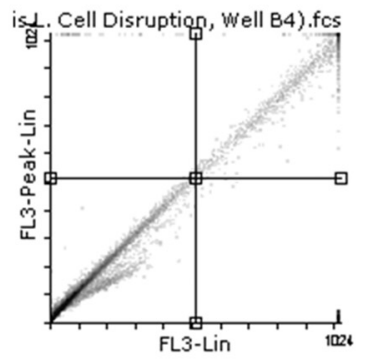

f

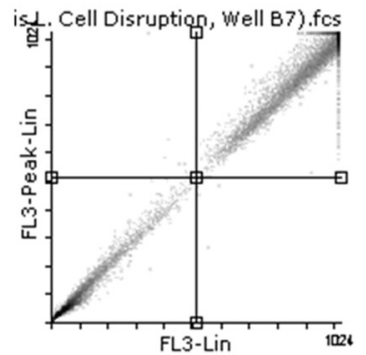

$\mathbf{g}$

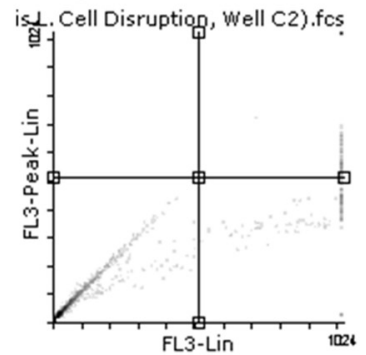

Fig. 2. Fluorescence profile of $M$. aeruginosa cells present in solution after applying the optimal disruption conditions selected for the different techniques tested. a) control; b) HSH; c) bead milling; d) microwave; e) FTC; f) sonication; and g) FTC followed by sonication.

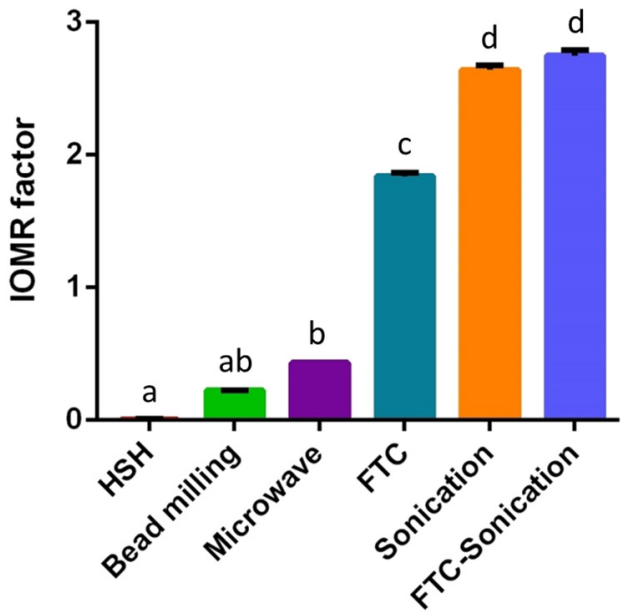

Fig. 3. Evaluation of IOMR of M. aeruginosa cells under the optimized conditions of each disruption method. Error bars represent the standard deviation for three experiments. According to Tukey's test, the methods presenting the same letter do not differ significantly $(\alpha=0.05)$.

most effective techniques with regards to cells' viability reduction (Fig. 1). The HSH method, in turn, had no effect on IOMR (Fig. 3), which was already expected since it did not show to induce any significant changes in cells' structure (Fig. 1 and Fig. 2). Despite of the greater IOMR derived from sonication treatment, and as discussed in the previous section, this methodology is frequently taken as unsuitable for extraction purposes. As example, Lee et al. [37] determined that the extraction of lipids involving three different microalgae (C. vulgaris, Botrycoccus sp., and Scenedesmus sp.) is more efficient using microwave irradiation, followed by bead-beating and sonication, respectively. The same conclusions were drawn by Heo et al. [36] when comparing the extraction of lipids from $C$. vulgaris using microwave and sonication, with efficiencies of 82.87 and $69.56 \%$, respectively. Differently, Pan et al. [38] have found similar extraction yields of bioactive compounds from a plant by applying microwave irradiation and sonication. In addition to the utilization of different organisms, the variability observed between our results and the other studies might be explained by the use of different disruption conditions of the tested methods and the application of solvents to enhance the extraction of certain compounds.

\subsubsection{Toxin release}

As displayed in Fig. 4, the release of MC from M. aeruginosa cells was also evaluated for each optimized disruption process.

Despite of the lower impact on cells' viability when compared to microwave, FTC, and the combination with FTC (Fig. 1), sonication was, together with the combination with FTC, the most successful methodology with respect to toxin release, similarly to what was observed for the IOMR (Fig. 3). On the contrary, HSH and bead milling, that generally presented none and intermediate impact on $M$. aeruginosa cells (cf. Sections 3.2.1 and 3.2.2), respectively, do not seem to be interesting methods to conduct MC's release processes, once no significant changes were determined when compared to control. However, bead milling also demonstrated to be statistically identical to microwave, FTC, and sonication followed by FTC, which allows us to conclude that

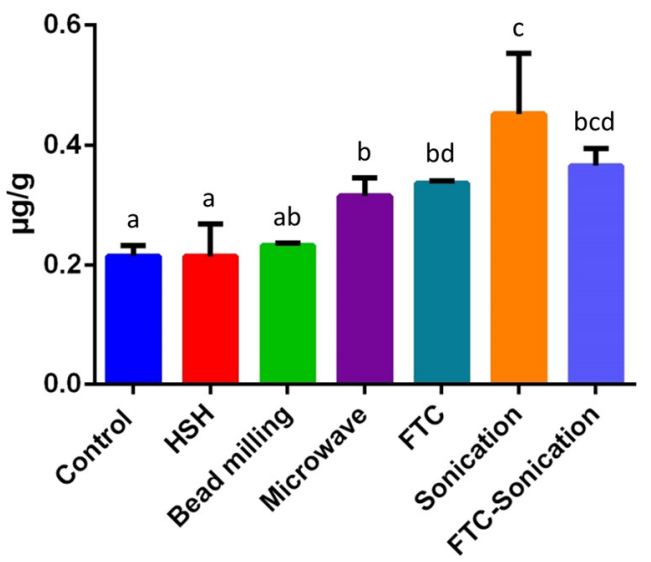

Fig. 4. MC release assessment of $M$. aeruginosa cells under the optimized conditions of each disruption method. Error bars represent the standard deviation for three experiments. According to Tukey's test, the methods presenting the same letter do not differ significantly $(\alpha=0.05)$. 
this method is not very efficient on MC release but, at the same time, it is not as inefficient as HSH. This distinction is clearly supported by the results obtained for cells' viability (Fig. 1) and IOMR (Fig. 3) where the impact of HSH is neglectable, while bead milling has shown to affect $M$. aeruginosa in both parameters. Regarding microwave and FTC, these techniques were not able to reach a toxin release significantly different from that observed with FTC followed by sonication method. The similarities between these three methodologies could be expected taking into account the impact of all of them over cells (Fig. 1); however, it is interesting to see that microwave irradiation and FTC did not present comparable yields of IOMR (Fig. 3) and MC release (Fig. 4) to sonication, which revealed lower reduction on cells' viability. Even more surprising is the fact that FTC is pointed by the manufacturers' instructions of most of the toxin quantification ELISA kits (including the one used in our study) as the disruption method to apply. Fig. 4 clearly shows that FTC is not the best option to promote the release of MC and, when applied as pre-treatment of sonication, no increment was noted.

These results are not in agreement with Silva-Stenico et al. [16], once microwave and sonication approaches revealed similar toxin release yields. Additionally, the amount of MC obtained by these authors corresponds to concentrations 1000 times higher than ours, which is very surprising. The hypotheses for such huge differences might be the use of distinct strains, which have certainly different toxin production capacities and probably different resistance to disruption methods, or the use of different treatment conditions. For instance, the microwave irradiation process described by Silva-Stenico et al. [16] was applied for a longer period of time ( $15 \mathrm{~min}$ ) and the solution was boiled, being thus more aggressive to cells than ours $(1.5 \mathrm{~min}$ of treatment divided into $15 \mathrm{~s}$ cycles and temperatures always below $60^{\circ} \mathrm{C}$ throughout the whole process). Furthermore, in the aforementioned research work, all the samples undergone disruption processing twice since the treatments were repeated and re-extraction was performed, which might have led to this exponential release of MC. Additionally, the cells used by SilvaStenico and her colleagues [16] were previously lyophilized and that might have weakened the cells' membranes before applying the disruption methods, contributing to higher amounts of toxin extracted.

\section{Conclusions}

Each disruption technique was optimized through the assessment of IOMR and the most suitable conditions were defined as follows: i) $20 \%$ of beads and treatment time of $7 \mathrm{~min}$ (bead milling); ii) $800 \mathrm{~W}$ for $1.5 \mathrm{~min}$ (microwave); iii) three $12 \mathrm{~h}$ freezing cycles at $-20{ }^{\circ} \mathrm{C}$ (FTC); iv) $15,000 \mathrm{rpm}$ for $7 \mathrm{~min}$ (HSH); and v) $40 \mathrm{kHz}$ for $10 \mathrm{~min}$ (sonication). Among the optimized methodologies, HSH revealed to be ineffective to extract the toxin or any other intracellular matter, which is a consequence of the absence of structural alterations inflicted to cells. In contrast, FTC followed by sonication, FTC, and microwave irradiation had a great impact over $M$. aeruginosa cells and all of them presented $<1 \%$ of viable cells after treatment application. However, cell damage was not accompanied by the values of IOMR and MC release in the case of FTC and microwave. Sonication, which was not as effective in reducing cells' viability $-5 \%$ of the initial biomass concentration maintained its viability, has shown to be the best methodology to apply both for IOMR and toxin release, together with FTC followed by sonication. Generally, bead milling has demonstrated a low to intermediate effect on MC release and IOMR but was able to reduce the cells' viability in $84.5 \%$.

\section{Acknowledgments}

This research work was supported by the grant SFRH/BPD/98694/ 2013 (Bruno Fernandes) and SFRH/BD/52335/2013 (Pedro Geada) from Fundação para a Ciência e a Tecnologia (Portugal). Luís Loureiro is recipient of a fellowship supported by a doctoral advanced training (call NORTE-69-2015-15) funded by the European Social Fund under the scope of Norte2020 - Programa Operacional Regional do Norte. This study was supported by the Portuguese Foundation for Science and Technology (FCT) under the scope of the strategic funding of UID/BIO/ 04469/2013 unit and COMPETE 2020 (POCI-01-0145-FEDER-006684), Project UID/Multi/04423/2013, Project RECI/BBB-EBI/0179/2012 (FCOMP-01-0124-FEDER-027462), FCT Strategic Project of UID/BIO/ 04469/2013 unit, by the project NOVELMAR (reference NORTE-010145-FEDER-000035), co-financed by the North Portugal Regional Operational Programme (Norte 2020) under the National Strategic Reference Framework (NSRF), through the ERDF, and by BioTecNorte operation (NORTE-01-0145-FEDER-000004) funded by the European Regional Development Fund under the scope of Norte2020 — Programa Operacional Regional do Norte.

\section{Declaration of authors' contribution}

Conception and design of study: P. Geada and B.D. Fernandes; Acquisition of data: P. Geada and L. Loureiro; Analysis and/or interpretation of data: P. Geada, L. Loureiro, J.A. Teixeira, V. Vasconcelos, A.A. Vicente, and B.D. Fernandes; Drafting the manuscript: P. Geada and B.D. Fernandes; Revising the manuscript critically for important intellectual content: P. Geada, L. Loureiro, J.A. Teixeira, V. Vasconcelos, A.A. Vicente, and B.D. Fernandes; Approval of the version of the manuscript to be published: P. Geada, L. Loureiro, J.A. Teixeira, V. Vasconcelos, A.A. Vicente, and B.D. Fernandes.

\section{Statement of informed consent, human/animal rights}

No conflicts, informed consent, or human/animal rights are applicable to this study.

\section{References}

[1] A. Oren, Chapter 1 - cyanobacteria: biology, ecology and evolution, in: N.K. Sharma, A.K. Rai, L.J. Stal (Eds.), Cyanobacteria: An Economic Perspective, Jonh Willey \& Sons, Ltd, UK, 2014, pp. 1-20.

[2] J.M. O'Neil, T.W. Davis, M.A. Burford, C.J. Gobler, The rise of harmful cyanobacteria blooms: the potential roles of eutrophication and climate change, Harmful Algae 14 (2012) 313-334.

[3] P. Geada, S. Gkelis, J. Teixeira, V. Vasconcelos, A. Vicente, B. Fernandes, Chapter 17: cyanobacterial toxins as a high added-value product, in: R. Muñoz, C. Gonzalez (Eds.), Microalgae-based Biofuels and Bioproducts, Woodhead Publishing, UK, 2017, pp. 405-432.

[4] N. Thajuddin, G. Subramanian, Cyanobacterial biodiversity and potential applications in biotechnology, Curr. Sci. 89 (2005) 47-57.

[5] L. Li, N. Gao, Y. Deng, J. Yao, K. Zhang, Characterization of intracellular and extracellular algae organic matters (AOM) of Microcystis aeruginosa and formation of AOM-associated disinfection byproducts and odor and taste compounds, Water Res. 46 (2012) 1233-1240.

[6] E.M. Jochimsen, W.W. Carmichael, J. An, D.M. Cardo, S.T. Cookson, C.E.M. Holmes, M.B.C. Antunes, D.A.M. Filho, T.M. Lyra, V.S.T. Barreto, S.M.F.O. Azevedo, W.E. Jarvis, Liver failure and death after exposure to microcystins at a hemodialysis center in Brazil, N. Engl. J. Med. 338 (1998) 873-878.

[7] V. Ashokkumar, E. Agila, Z. Salam, M. Ponraj, M.F.M. Din, F.N. Ani, A study on large scale cultivation of Microcystis aeruginosa under open raceway pond at semicontinuous mode for biodiesel production, Bioresour. Technol. 172 (2014) 186-193.

[8] F. Chen, H.-B. Li, R.N.-S. Wong, B. Ji, Y. Jiang, Isolation and purification of the bioactive carotenoid zeaxanthin from the microalga Microcystis aeruginosa by highspeed counter-current chromatography, J. Chromatogr. A 1064 (2005) 183-186.

[9] R. Philippis, M. Vincenzini, Exocellular polysaccharides from cyanobacteria and their possible applications, FEMS Microbiol. Rev. 22 (1998) 151-175.

[10] P.C.M. Rós, C.S.P. Silva, M.E. Silva-Stenico, M.F. Fiore, H.F. Castro, Microcystis aeruginosa lipids as feedstock for biodiesel synthesis by enzymatic route, J. Mol, Catal. B Enzym. 84 (2012) 177-182.

[11] S.P. Singh, P. Singh, Effect of $\mathrm{CO}_{2}$ concentration on algal growth: a review, Renew. Sust. Energ. Rev. 38 (2014) 172-179.

[12] E. Günerken, E. D'Hondt, M.H.M. Eppink, L. Garcia-Gonzalez, K. Elst, R.H. Wijffels, Cell disruption for microalgae biorefineries, Biotechnol. Adv. 33 (2015) 243-260.

[13] M. Vanthoor-Koopmans, R.H. Wijffels, M.J. Barbosa, M.H.M. Eppink, Biorefinery of microalgae for food and fuel, Bioresour. Technol. 135 (2013) 142-149.

[14] M. Ma, R. Liu, H. Liu, J. Qu, Chlorination of Microcystis aeruginosa suspension: cell lysis, toxin release and degradation, J. Hazard. Mater. 217-218 (2012) 279-285.

[15] C.J. Pestana, P.J. Reeve, G. Newcombe, Extraction method for total microcystins in cyanobacteria-laden sludge, J. Chromatogr. B 965 (2014) 61-64. 
[16] M.E. Silva-Stenico, R.C. Neto, I.R. Alves, L.A.B. Moraes, T.K. Shishido, M.F. Fiore, Hepatotoxin microcystin-LR extraction optimization, J. Brazil. Chem. Soc. 20 (2009) 535-542.

[17] X. Wu, E.M. Joyce, T.J. Mason, Evaluation of the mechanisms of the effect of ultrasound on Microcystis aeruginosa at different ultrasonic frequencies, Water Res. 46 (2012) 2851-2858.

[18] J. Kotai, Instructions for Preparation of Modified Nutrient Solution Z8 for Algae, Norwegian Institute for Water Research, Blindern, Oslo, 1972, p. 5.

[19] K.I. Harada, K. Tsuji, M.F. Watanabe, F. Kondo, Stability of microcystins from cyanobacteria III. Effect of pH and temperature, Phycologia 35 (1996) 83-88.

[20] World Health Organization, Cyanobacterial Toxins: Microcystin-LR in Drinking Water, Background Document for Preparation of WHO Guidelines for Drinking Water Quality, (2003).

[21] E. Günerken, E. D'Hondt, M. Eppink, K. Elst, R. Wijffels, Flow cytometry to estimate the cell disruption yield and biomass release of Chlorella sp. during bead milling, Algal Res. 25 (2017) 25-31.

[22] R.K. Henderson, A. Baker, S.A. Parsons, B. Jefferson, Characterisation of algogenic organic matter extracted from cyanobacteria, green algae and diatoms, Water Res. 42 (2008) 3435-3445.

[23] A. Richmond, Handbook of Microalgal Culture: Biotechnology and Applied Phycology, Blackwell Publishing Ltd, UK, 2004.

[24] J. Doucha, K. Lívanský, Influence of processing parameters on disintegration of Chlorella cells in various types of homogenizers, Appl. Microbiol. Biotechnol. 81 (2008) 431-440.

[25] V. Montalescot, T. Rinaldi, R. Touchard, S. Jubeau, M. Frappart, P. Jaouen, P. Bourseau, L. Marchal, Optimization of bead milling parameters for the cell disruption of microalgae: process modeling and application to Porphyridium cruentum and Nannochloropsis oculata, Bioresour. Technol. 196 (2015) 339-346.

[26] A.R. Byreddy, C.J. Barrow, M. Puri, Bead milling for lipid recovery from thraustochytrid cells and selective hydrolysis of Schizochytrium DT3 oil using lipase, Bioresour. Technol. 200 (2016) 464-469.

[27] P.R. Postma, T.L. Miron, G. Olivieri, M.J. Barbosa, R.H. Wijffels, M.H.M. Eppink, Mild disintegration of the green microalgae Chlorella vulgaris using bead milling,
Bioresour. Technol. 184 (2015) 297-304.

[28] S. Balasubramanian, J.D. Allen, A. Kanitkar, D. Boldor, Oil extraction from Scenedesmus obliquus using a continuous microwave system — design, optimization, and quality characterization, Bioresour. Technol. 102 (2011) 3396-3403.

[29] J.R. McMillan, I.A. Watson, M. Ali, W. Jaafar, Evaluation and comparison of algal cell disruption methods: microwave, waterbath, blender, ultrasonic and laser treatment, Appl. Energy 103 (2013) 128-134.

[30] A.C. Guedes, H.M. Amaro, M.S. Gião, F.X. Malcata, Optimization of ABTS radical cation assay specifically for determination of antioxidant capacity of intracellular extracts of microalgae and cyanobacteria, Food Chem. 138 (2013) 638-643.

[31] G. Wang, T. Wang, Characterization of lipid components in two microalgae for biofuel application, J. Am. Oil Chem. Soc. 89 (2012) 135-143.

[32] H. Sun, B. Guan, Q. Kong, Z. Geng, N. Wang, Repeated cultivation: non-cell disruption extraction of astaxanthin for Haematococcus pluvialis, Sci. Rep. 6 (2016) 20578.

[33] A. Richmond, Q. Hu, Handbook of Microalgal Culture: Applied Phycology and Biotechnology, John Wiley \& Sons, Ltd, UK, 2013.

[34] M. Wang, W. Yuan, Microalgal cell disruption in a high-power ultrasonic flow system, Bioresour. Technol. 193 (2015) 171-177.

[35] O. Parniakov, E. Apicella, M. Koubaa, F.J. Barba, N. Grimi, N. Lebovka, G. Pataro, G. Ferrari, E. Vorobiev, Ultrasound-assisted green solvent extraction of high-added value compounds from microalgae Nannochloropsis spp. Bioresour. Technol. 198 (2015) 262-267.

[36] Y.M. Heo, H. Lee, C. Lee, J. Kang, J.-W. Ahn, Y.M. Lee, K.-Y. Kang, Y.-E. Choi, J.J. Kim, An integrative process for obtaining lipids and glucose from Chlorella vulgaris biomass with a single treatment of cell disruption, Algal Res. 27 (2017) 286-294.

[37] J.-Y. Lee, C. Yoo, S.-Y. Jun, C.-Y. Ahn, H.-M. Oh, Comparison of several methods for effective lipid extraction from microalgae, Bioresour. Technol. 101 (2010) S75-S77.

[38] X. Pan, G. Niu, H. Liu, Comparison of microwave-assisted extraction and conventional extraction techniques for the extraction of tanshinones from Salvia miltiorrhiza bunge, Biochem. Eng. J. 12 (2002) 71-77. 